\title{
Comparison of surface microfouling and bacterial attachment on the egg capsules of two molluscan species representing Cephalopoda and Neogastropoda
}

\author{
Norman S. H. Lim, Kim J. Everuss, Amanda E. Goodman, Kirsten Benkendorff* \\ School of Biological Sciences, Flinders University of South Australia, GPO Box 2100, Adelaide, \\ South Australia 5001, Australia
}

\begin{abstract}
Many organisms naturally defend themselves against microbial attachment and biofouling in the marine environment. In this study, we investigated microbial fouling on 2 molluscan egg capsules using scanning electron microscopy (SEM), two-photon laser scanning microscopy (TPLSM) with bacterial viability staining and bacterial attachment experiments with the biofilm-forming Pseudoalteromonas sp. S91 in flow chambers. Results indicated that early stage egg capsules of Dicathais orbita (Neogastropoda) are relatively free of surface microorganisms. Egg capsules during the trocophore stage had a regularly ridged microtexture, but as capsules matured, shedding of the outer wall was observed, followed by the extrusion of unidentified droplets, which then accumulated on the capsule surface in association with bacteria. By comparison, the egg capsules of Sepioteuthis australis (Cephalopoda) were found to have an irregular surface with many hills and valleys that accommodate colonization by a variety of microorganisms. At the later stages of development these squid egg capsules become heavily colonized by algal spores. Cross sections of egg capsules revealed that $S$. australis capsule walls were about 12 times thicker than $D$. orbita egg capsules. Staining the egg capsules with BacLight ${ }^{\mathrm{TM}}$ also revealed a significantly thicker biofilm, with more live and dead bacteria on $S$. australis capsules than on those of $D$. orbita $(\mathrm{p}<0.05)$. Flow chamber experiments indicated that the surface of $S$. australis capsules provided a suitable substrate for colonization by Pseudoalteromonas sp. S91, whereas colonization was significantly less on $D$. orbita egg capsules after 24 and $72 \mathrm{~h}(\mathrm{p}<0.01)$. These experiments indicated that $D$. orbita egg capsules are better defended against fouling microbes than are the eggs of $S$. australis. $D$. orbita appears to use a combination of physical, mechanical and possibly chemical defense mechanisms to reduce fouling on their egg capsules.
\end{abstract}

KEY WORDS: Biofilm · Egg capsules $\cdot$ Mollusc $\cdot$ Scanning electron microscopy $\cdot$ Two-photon laser scanning microscopy $\cdot$ Bacterial attachment

Resale or republication not permitted without written consent of the publisher

\section{INTRODUCTION}

The economic costs of biofouling on artificial structures, such as boat hulls, nets and pipes, can be enormous. This has resulted in the use of toxic paints that have led to serious environmental problems (e.g. tributyltin, TBT; Alzieu 2000, Champ 2000, Goldberg et al. 2004). Biofilm formation also plays an important role in bacterial pathogenesis. Biofilms are typically resistant to antibiotics, providing a source for persistent infec- tion (Hentzer \& Givskov 2003) and/or re-infection from medical tubing and equipment. Understanding the mechanisms by which aquatic organisms naturally regulate fouling of their surfaces should provide a platform for ongoing developments in the control of economically and ecologically important fouling processes (de Nys \& Steinburg 2002).

Surface fouling by microorganisms is one of the many threats that face organisms in the marine environment (Davis et al. 1989, Scardino et al. 2003). With 
an estimated density of $5 \times 10^{5}$ prokaryotic cells $\mathrm{ml}^{-1}$ seawater (Whitman et al. 1998), sessile invertebrates and algae are exposed to a constant onslaught of potentially detrimental microbes. These include biofilm-forming bacteria along with single-cell diatoms that rapidly settle, attach and form colonies on any surface placed in the marine environment (Davis et al. 1989). The formation of a microbial biofilm promotes the attachment of algal spores, protozoa, barnacle cyprids and marine fungi, followed by the settlement of other marine invertebrate larvae and macroalgae (Maki 2000, Callow \& Callow 2002). Heavy surface fouling could lead to the accumulation of toxic wastes, a reduction in oxygen and nutrient availability and increased drag, which can cause sessile organisms to become dislodged from benthic substrata in strong currents. All extant marine organisms have survived for millions of years under the significant selective pressure of surface fouling and therefore must have evolved successful mechanisms for reducing these negative impacts.

The encapsulation of developing offspring within elaborate structures that are deposited on benthic substrata is a common reproductive strategy among marine molluscs. These egg capsules appear to be highly resilient multilaminate biomaterials (Rapoport \& Shadwick 2002) that require substantial maternal energy investment (Stickle 1973). This implies that egg capsules must provide adaptive benefits that increase embryonic survivorship. The functional role of encapsulated development is widely attributed to the protection of developing offspring from environmental stresses, as well as to deter potential predators and pathogens (Thorson 1950, Pechenik 1986, Rawlings 1999, Przeslawski 2004). However, these egg capsules can remain in the marine environment for several months, and thus would also be vulnerable to surface fouling. Nevertheless, previous studies indicate that these egg capsules remain axenic (Lord 1986) and remarkably free of surface macrofouling (Przeslawski \& Benkendorff 2005). Antimicrobial activity also appears to be widespread in chemical extracts from molluscan egg masses (Benkendorff et al. 2001, Santhana Ramasamy \& Murugan 2005), but it is presently uncertain if the active compounds are located on the eggs' surface. Interestingly, a previous study on the egg cases of the dogfish Scyliorhinus canicula demonstrated that these marine egg capsules are resistant to microbial degradation and performed much better than many commercial antifouling treatments in the field (Thomason et al. 1994). Mechanisms for deterring fouling on these extracellular structures are not currently understood.

The functional mechanisms for preventing detrimental microbial colonization on marine organisms can include associated chemical, mechanical and/or physical defenses. Chemical extracts from a wide variety of marine invertebrates and algae have demonstrated antimicrobial activity against marine biofilm bacteria (Steinberg et al. 2001, Engel et al. 2002, Lindquist 2002, Santhana Ramasamy \& Murugan 2005) and some secondary metabolites have been shown to regulate biofilm bacteria on biotic surfaces. For example, furanones produced by the red alga Delisea pulchra actively prevent bacterial and larval settlement (Dworjanyn et al. 1999, Steinberg et al. 2001). Secondary metabolites produced by sponges in the Caribbean were also shown to prevent bacterial surface colonization (Kelly et al. 2005). Mechanical methods of fouling prevention are adopted by the macroalga Dilsea carnosa, which sheds its epidermis and cuticle to remove any epibionts (Nylund \& Pavia 2005). Similarly, moulting in crustaceans would facilitate the removal of epibionts (Dyrynda 1986). Relatively little is known of the role of physical defenses (Steinberg et al. 2001), though surface composition and microtexture can influence the rate of biofouling (Berntsson et al. 2000, Steinberg \& De Nys 2002, Baum et al. 2003).

Biofilms cultivated on inanimate and biological surfaces under laboratory conditions have been studied extensively using a wide range of microscopic techniques (Beech et al. 2000), often in combination, to give a clear description of the biofilm. Scanning electron microscopy (SEM) allows morphological examination of biofilms established on surfaces (Beech et al. 2000). Bers \& Wahl (2004) used SEM to study the surface properties of different marine organisms, such as the edible crab Cancer pagurus (Crustacea), the blue mussel Mytilus edulis (Bivalvia), the brittle star Ophinura texturata (Ophiuroidea) and the egg case of the lesserspotted dogfish Scyliorhinus canicula (Chondrichthyes), which are all seldom associated with epibionts. These studies support the evolution of physical antifouling strategies, showing that homogeneous surfaces are capable of deterring attachment by limiting space available for fouling organisms.

Flow chambers such as those designed by Hale \& Mitchell (2001) can also be used to study biofilm formation. Bacterial attachment to biological surfaces can be studied using Pseudoalteromonas sp. S91, a motile Gram-negative marine bacterium that secretes hydrolytic enzymes, e.g. chitinases and proteases (Techkarnjanaruk \& Goodman 1999), and can utilize squid pen as a nutrient source (Techkarnjanaruk et al. 1997). The derivative strain, S91SB, has a transposon insert containing a copy of the green fluorescent protein (GFP) gene originated from the jellyfish Aequoria victoria (Chalfie et al. 1994). The use of GFP in prokaryotes has assisted the study of spatial distribution of microbial cells within a biofilm (Yoshida \& 
Kuramitsu 2002). In addition, the use of GFP in conjunction with two-photon laser scanning microscopy (TPLSM) can facilitate the real-time analysis of cells within a living biofilm (Stretton et al. 1998).

This study examines microbial fouling on the surface of egg capsules from 2 molluscan species: the white dogwhelk Dicathais orbita (Neogastropoda: Muricidae) and the southern calamari Sepioteuthis australis (Cephalopoda: Loliginidae) using a combination of microscopic techniques. The recent study by Przeslawski \& Benkendorff (2005) on the egg masses of 18 gastropod molluscs revealed that the leathery egg capsules of neogastropods, including $D$. orbita, were significantly less fouled than a range of gelatinous egg masses. However, in Przeslawski \& Benkendorff's (2005) study, protists and algal fouling were only estimated visually under a dissecting microscope. Similarly, Steer et al. (2002) used visual observations to report high levels of algal fouling on the surface of egg capsules of $S$. australis. These preliminary results suggested that egg capsules from different molluscan species are differentially protected from surface fouling, but further investigation is required to reveal the underlying mechanisms. Therefore, the aim of the present study was to compare the physical structure and how this influences the biofouling processes on the surface of egg capsules from a cephalopod and neogastropod. We used SEM to study the surface morphology of the egg capsules, as well as the density and types of microorganisms present on the surface of capsules at different stages of embryonic development. The LIVE/DEAD ${ }^{\circledR}$ BacLight $^{\mathrm{TM}}$ bacterial viability assay was used to quantify the amount of live and dead bacteria on the surface. In addition, bacterial attachment experiments were conducted using Pseudoalteromonas sp. S91SB in flow chambers and TPLSM to quantify bacterial accumulation on the surface of egg capsules.

\section{MATERIALS AND METHODS}

Collection of Dicathais orbita and Sepioteuthis australis egg capsules. The egg masses of $D$. orbita were collected from the lower intertidal regions of rocky reefs along the Fleurieu Peninsula, South Australia, from early spring (October) to mid-summer (December) 2005. Egg capsules of $S$. australis were obtained from subtidal seagrass habitats at Glenelg, South Australia, and stored at $4{ }^{\circ} \mathrm{C}$ to prevent deterioration. Egg capsules of both species were separated into different stages of development: the fresh (undifferentiated embryos), trocophore (ciliated embryos with no shell) and developed (veligers with pigmented shell) stages of $D$. orbita and the fresh (undifferentiated embryos) and developed (paralarvae) stages of $S$. australis were examined. Identification of developmental stage was performed by dissecting capsules from each replicate egg cluster and examining the contents under a dissecting microscope.

Dicathais orbita capsules of known age since deposition were also collected from a recirculating seawater system at Flinders University to allow SEM images of veliger stage to be separated into 3,4 and $>4$ wk age classes. However, for the quantification of fouling on the surface and in all other experiments, egg capsules were collected from the field (exact age uncertain) to ensure representation of the natural status. For the live/dead bacterial staining and the bacterial attachment experiments, $D$. orbita egg capsules were used in the trocophore stage (owing to interesting microtopography observed at this stage-see 'Results; Scanning electron micrography'), and fresh squid egg capsules with undifferentiated embryos were used because these are much closer in age to the D. orbita trocophores than are the paralarvae.

Scanning electron microscopy. For each species, 5 independent egg capsules at each stage of embryonic development were fixed overnight in electron microscope fixative $(1.25 \%$ glutaraldehyde, $4 \%$ sucrose, $4 \%$ paraformaldehyde in phosphate buffered saline [PBS], $\mathrm{pH}$ 7.2). After $24 \mathrm{~h}$, the egg capsules were removed from fixative, washed twice with PBS and cut into $0.5 \times$ $0.5 \mathrm{~cm}$ sections. Samples were then fixed in $2 \%$ osmium tetroxide for $60 \mathrm{~min}$ before dehydration in increasing concentrations of ethanol $(70,90,95$ and $100 \%$ for $30 \mathrm{~min}$ each). Finally, the samples were dried under pressure with liquid carbon dioxide in a BalTec CPD030 Critical Point Dryer. The dried samples were placed on metal stubs using adhesive stickers and sputter coated with platinum. Egg capsules were orientated to allow morphological examination and analysis of surface bacterial load. Cross-sectional views of egg capsules were also examined. The samples were viewed using a Philips XL-30 Field Emission SEM.

Cryogenic SEM was also carried out using an Oxford Instruments CT1500 HF Cryo Preparation System attached to the SEM. Egg capsules were attached to the holder using Tissue-Tek OCT compound, then frozen in nitrogen slush, and transferred under vacuum to the preparation chamber. The temperature of the sample was raised to $-92^{\circ} \mathrm{C}$ and held there for approximately $3 \mathrm{~min}$ to allow any ice on the surface to sublime. After lowering the temperature to $-110^{\circ} \mathrm{C}$ to halt sublimation, the sample was coated with platinum, loaded onto the microscope stage and examined whilst being maintained at $-150^{\circ} \mathrm{C}$.

The percentage of fouling on the surface of egg capsules was determined using images taken from SEM analysis. For each egg capsule, 5 SEM images were 
examined at approximately 5000× magnification, and the percentage cover per image was estimated visually and then averaged to obtain 1 mean value per capsule. A 2-factor ANOVA was performed using the statistical package SPSS version 11.0 to determine if species and stage of development (freshly laid or developed) affected surface fouling. A separate 1-way ANOVA was also performed on Dicathais orbita (fresh, trocophore and late stage veliger) egg capsule images to determine if the extent of fouling at the trocophore stage differed from the other developmental stages. The assumption of equal variances was tested using Levene's test and normality was tested using the Kolmogorov-Smirnov test in SPSS. The $\alpha$-level was set at 0.05 for a significant difference, but then Bonferroni corrected to 0.0167 to account for the multiple comparisons using the same data (Quinn \& Keough 2002).

LIVE/DEAD BacLight bacterial viability assay. A stock solution was prepared by mixing equal volumes of the reagents SYTO 9 and propidium iodide (Invitrogen, see product information, www.invitrogen.com). The working solution was then prepared by adding $3 \mu \mathrm{l}$ of the stock solution to $1 \mathrm{ml}$ of $0.85 \% \mathrm{NaCl}$. Positive and negative controls of live and ethanol-killed Pseudoalteromonas sp. S91SB cultures were independently stained to optimize the concentration of dyes and parameters for Confocal Laser Scanning Microscopy (CLSM).

Six independent capsules from each species were stained and examined. Under sterile conditions, egg capsules were excised from the cluster and rinsed with Milli-Q water before incubation in the working dye solution for $15 \mathrm{~min}$ in the dark. After incubation, a medial section of the egg capsule was prepared using sterile forceps and a surgical blade. The apex and basal regions of the egg capsule were removed, and a section of capsule wall $(\sim 0.5 \times 0.5 \mathrm{~cm})$ mounted onto a clean glass slide and viewed under an Olympus BX50F-3 epifluorescence microscope. A Bio-Rad MRC-1000UV CLSM was subsequently used to capture quality images of the stained egg capsules. Images in the green spectra (live) and red spectra (dead) were taken separately for quantification purposes.

The amount of live and dead microorganisms was quantified using Image $\mathrm{J}$ 1.32j software. The total area of fluorescence within $x z$ images was quantified under a standardized area of $96.26 \mu \mathrm{m}(x)$ by $96.26 \mu \mathrm{m}(z)$. Paired sample $t$-tests were used to compare the amount of green and red fluorescence on the surface of egg capsules within each species $(n=6)$. One-way ANOVAs were then used to separately compare the amount of green and red fluorescence between species. A square-root transformation was performed on the data in order to meet the assumptions of equal variances (Levene's test). After applying a Bonferroni correction, $\alpha$ was set at 0.0167 .

Bacterial preparation. Pseudoalteromonas sp. Strain S91SB (hereafter S91SB) is a derivitive of Strain S91 (Techarnjanaruk et al. 1997) into which a gfp gene has been inserted. S91SB was grown in $10 \mathrm{ml}$ of marine minimal medium (MMM; Östling et al. 1991) supplemented with $0.2 \%$ glutamate at $30^{\circ} \mathrm{C}$ and allowed to grow overnight prior to use. Growth of S91SB was screened under a $100 \mathrm{~W}$ quartz halogen lamp with an infrared and $280 \mathrm{~nm}$ band-pass filter to check for GFP expression (Andover, part no. FS10-50) (Stretton et al. 1998). Green fluorescence indicated that bacteria were growing healthily.

Bacterial attachment experiment using flow chambers. Flow chamber experiments were carried out in triplicate using independent samples for 0,24 or $72 \mathrm{~h}$. Egg capsules were sterilized by dipping into $70 \%$ ethanol for $10 \mathrm{~min}$, and then washed with sterile Milli$\mathrm{Q}$ water before sectioning into $0.5 \times 0.5 \mathrm{~cm}$ pieces under aseptic conditions. Examination of control samples under the Olympus BX50F-3 epifluorescence microscope confirmed that this procedure successfully destroyed the existing biofilm without structurally altering the egg capsule walls. A single piece of capsule was placed into a once-through flow chamber, with the exterior surface facing up. Flow chambers used in this experiment were as described by Hale \& Mitchell (2001), and experimental conditions were maintained as described by Fitch et al. (2002). Two round glass coverslips (viewing ports) were sealed together with a rubber O-ring mounted in a stainless steel scaffold. The O-ring contained 2 needles acting as inlet and outlet ports. A piece of egg capsule was placed between the coverslips before sealing the chamber.

After assembly of the flow chamber, an 8-roller ColePalmer Masterflex peristaltic pump (7521-57) was used to pump $70 \%$ ethanol through the system for $15 \mathrm{~min}$, followed by sterile MMM for $20 \mathrm{~min}$. After flushing the system with sterile MMM, the peristaltic pump was turned off and $3 \mathrm{ml}$ of a $10^{-1}$ dilution of S91SB overnight culture was introduced. The chambers were left for $1 \mathrm{~h}$ to allow settlement and attachment of S91SB to egg capsules. Flow was resumed after $1 \mathrm{~h}$. Room temperature and flow rate was maintained at $24^{\circ} \mathrm{C}$ and $1 \mathrm{ml} \mathrm{min}^{-1}$, respectively (Delpin et al. 2000). Because MMM does not contain any carbon source, egg capsules were the only organic material available for bacterial attachment. After the completion of each time point $(0,24$ or $72 \mathrm{~h})$, the chambers were disconnected and taken to Adelaide Microscopy TPLSM for analysis.

Microscopic analysis of biofilm production using TPLSM. A Bio-Rad Radiance 2000MP in conjunction 
with a Nikon Eclipse TE300 inverted Multi-Photon Microscope was used to collect images of biofilms formed in the chambers. The microscope uses a $60 \times$ water immersion lens with a numerical aperture of 1.2 and a Coherent Mira900-F titanium:sapphire ultrafast laser, which has an excitation spectrum of pulsed $800 \mathrm{~nm}$ light equivalent to 1 photon of $400 \mathrm{~nm}$ light. GFP produced by S91SB was visualized using an excitation wavelength $(\lambda)$ of $800 \mathrm{~nm}$ and an emission $\lambda$ of $515 \mathrm{~nm}$. The 2 photons of $800 \mathrm{~nm}$ light are equivalent to $400 \mathrm{~nm}$ but do not damage cells as does $400 \mathrm{~nm}$ UV light typically used to excite GFP. Autofluorescence produced by the egg capsule was detected as blue at $460 \mathrm{~nm}$. Horizontal (xy) and vertical $(x z)$ optical sections of biofilm were imaged; within each $x y$ image, 3 random $x z$ sections were collected. This procedure was repeated 3 times for each chamber, yielding $3 x y$ and $9 x z$ images. Confocal Assistant was used to analyse all images and convert them to .tif files. Image J $1.32 \mathrm{j}$ was used to quantify the coverage of S91SB on egg capsules by calculating the total area of fluorescence, which is then divided by the mean area for a single S91SB cell $\left(0.56 \mu^{2}\right)$ (Everuss 2004). A standardized area of $x Z$ sections was used for quantification of fluorescence: $96 \mu \mathrm{m}(x)$ by $52 \mu \mathrm{m}(z)$.

One-way ANOVAs with Tukey's Honestly Significant Difference (HSD) post hoc tests were used to compare differences in the S91SB counts among each time point for Dicathais orbita and Sepioteuthis australis egg capsules. Independent $t$-tests were also used to compare the difference in S91SB counts between species at 24 and $72 \mathrm{~h}$. All analyses in this data set were tested more stringently ( $\alpha=0.01$ ) because assumptions of normality were not met (Underwood 1997), even after a series of transformations were attempted on the data (e.g. $\log x+1$, square root and cube root).

\section{RESULTS}

\section{Scanning electron microscopy}

The SEM revealed differences in the egg capsule structure of Dicathais orbita and Sepioteuthis australis capsules. Distinct layers of $D$. orbita egg capsules can be seen in transverse sections (Fig. 1a). The capsule comprises a solid inner wall and porous sections sandwiching a thick medial section with possible layering. The capsule wall is approximately $25 \mu \mathrm{m}$ thick (Fig. 1a). By comparison, S. australis capsules were observed to consist of multiple overlapping layers similar to a plywood structure (Fig. 1b). The capsule wall of fresh samples was approximately $300 \mu \mathrm{m}$ thick, and none of the layers appeared to be porous.
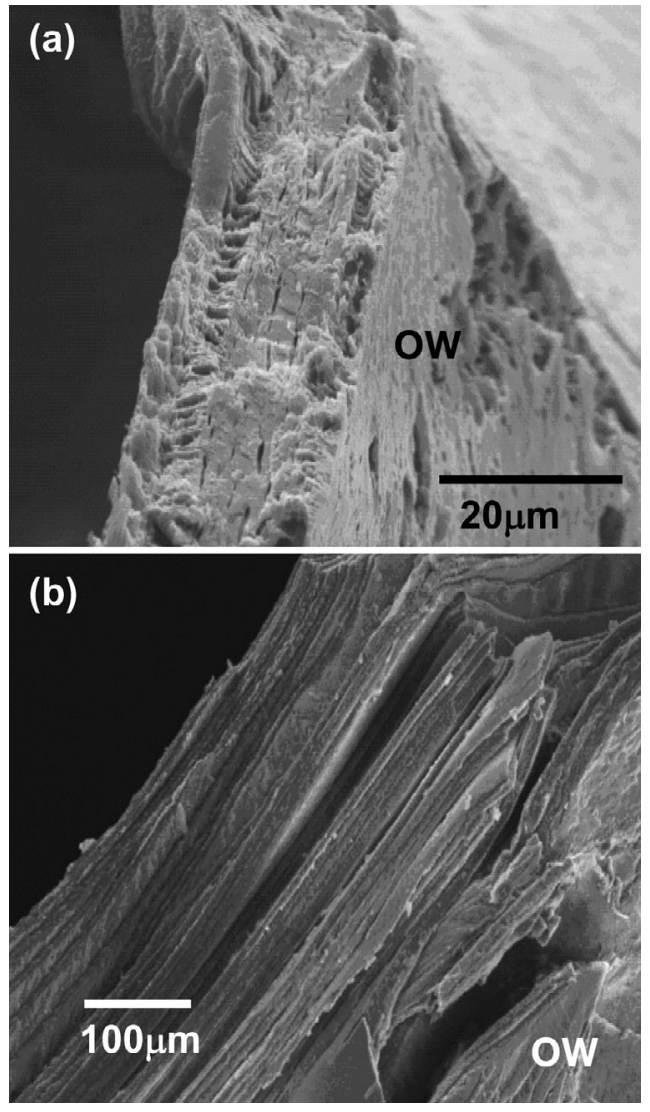

Fig. 1. (a) Dicathais orbita and (b) Sepioteuthis australis. Scanning electron micrographs showing transverse section of egg capsules. OW: outer wall

Differences in the surface microtopography were observed both between the species and within species at different stages of development (Figs. 2 \& 3). Freshly laid capsules (<1 wk old embryos) of Dicathais orbita possessed a thin layer of crust over a microtopographical structure on the outer-most surface (Fig. 2a). The surface was almost free from any bacteria and algae. As the egg capsules matured ( 1 to $3 \mathrm{wk}$ of age), the crust began to breakdown, exposing the microtopographical features (Fig. 2b). This layer has regular homogeneous ridges separated by 1 to $5 \mu \mathrm{m}$ (Fig. 2c). Sparse rod and cocci bacteria were observed on the walls and ridges during the second week of development. However, during the third week, densities of these bacteria increased and other fouling organisms such as filamentous algae were observed, forming a mixed biofilm community (Fig. 2d). In the later stages of development ( $>4$ wk old veligers), the fouled outer wall structure began to dissociate and shed from the capsule, leaving behind a naked capsule without any texture (Fig. 2e). This surface was initially free from microbes, but bacteria began colonization several days 

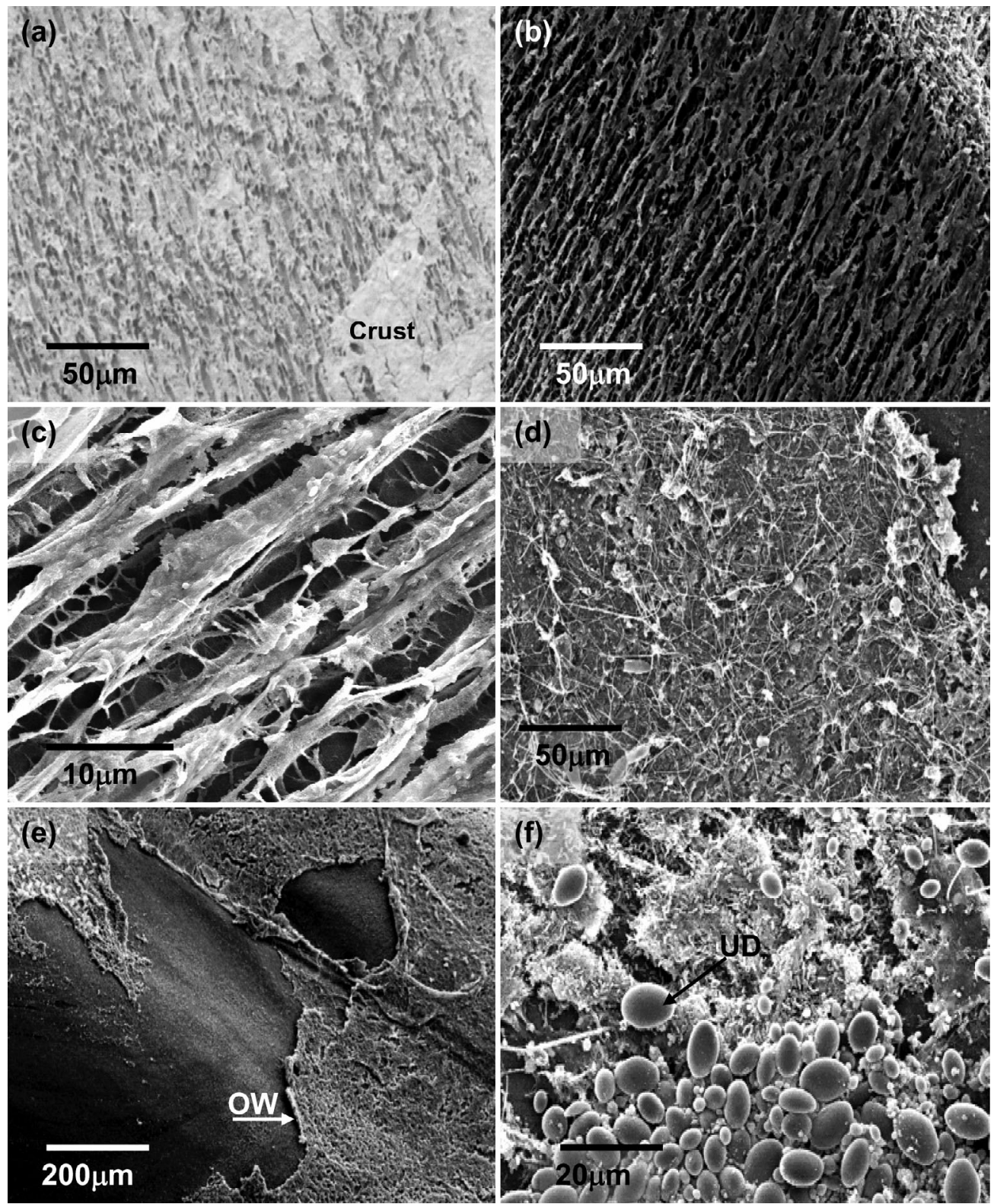

Fig. 2. Dicathais orbita. Scanning electron micrographs of the surface of egg capsules at different stages of development: (a) undifferentiated embryonic stage $<1 \mathrm{wk}$ old; (b) trocophore stage 1 to $3 \mathrm{wk}$ old; (c) trocophore stage showing microtopographical features; (d) veliger stage $>3 \mathrm{wk}$ old, showing increasing densities of microbial foulers; (e) veliger stage $>4$ wk old, with shedding of the fouled microtopographical structure (OW: outer wall); (f) late veliger stage $>4$ wk old, showing the presence of unidentified droplets (UD) on the surface

later. Unidentified droplets ranging from 8 to $20 \mu \mathrm{m}$ appeared on the surface of mature egg capsules after the majority of the outer wall dissociated (Fig. 2f). These droplets were either solitary or clumped in association with attached bacteria. A greater density of these droplets was observed on cryostat preparations than on critically point dried capsules. However, fewer microorganisms were observed on the surface of critically point dried samples (data not shown).
The surface of Sepioteuthis australis egg capsules typically had irregular folds resembling ridges and troughs about $5 \mu \mathrm{m}$ in width and depth (Fig. 3a). Freshly laid capsules (undifferentiated blastomere stage) collected from the sea contained fewer microorganisms on the surface (Fig. 3a,b) than did late-stage capsules containing paralarvae (Fig. 3c-f). Capsules of $S$. australis in the later stages of development collected from the field were covered with filamentous algae 

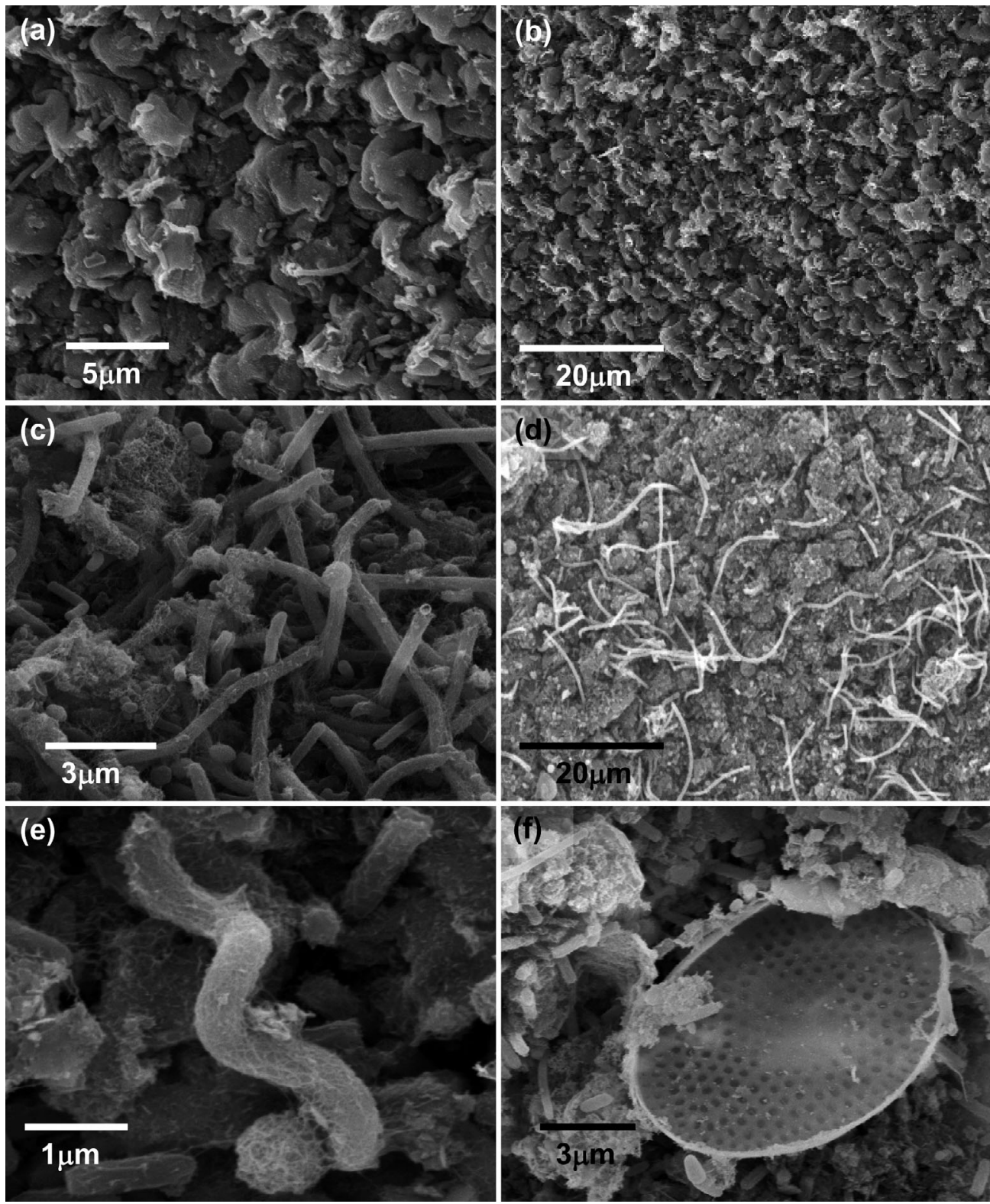

Fig. 3. Sepioteuthis australis. Scanning electron micrographs of the surface of egg capsules in 2 stages of development: (a,b) fresh capsules (undifferentiated embryos) showing complex microtopography; (c,d) heavily fouled capsules containing well-developed juveniles (paralarvae); (e) different bacteria and exopolymeric substances on the surface of a well-developed capsule; (f) a diatom on the surface of a well-developed capsule

and spores (Fig. 3c,d), as well as an exopolymeric substance and various bacteria (rod, cocci and spiral forms, e.g. Fig. 3e) and diatoms (Fig. 3f).

Fig. 4 shows the total percentage cover by all fouling organisms on the surface of Dicathais orbita and Sepioteuthis australis egg capsules. A 2-factor ANOVA was conducted to test the effect of species and stages of development (fresh and developed) on the level of fouling (percentage cover). Percentage coverage was significantly higher in the later stages of development (fresh embryos vs. developed capsules, $F=25.83, \mathrm{df}=$ $1, \mathrm{p}<0.001)$. However, species $(F=1.066, \mathrm{df}=1, \mathrm{p}=$ $0.317)$ and the interaction term $(F=2.296, \mathrm{df}=1, \mathrm{p}=$ 0.149 ) were not significant. A 1-way ANOVA for surface fouling on $D$. orbita capsules indicated that there was a statistically significant difference among the 3 different stages of development $(F=16.318, \mathrm{df}=2, \mathrm{p}<$ 0.001). Post hoc comparisons using Tukey's HSD test 


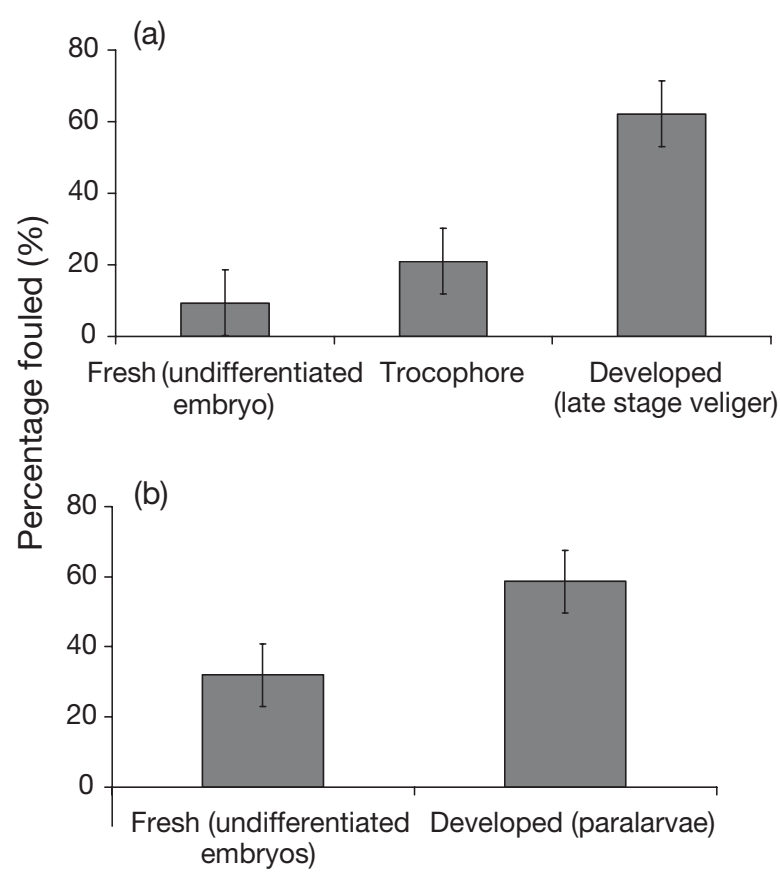

\section{Stage of development}

Fig. 4. (a) Dicathais orbita and (b) Sepioteuthis australis. Percentage surface covered by fouling organisms on molluscan egg capsules at different stages of development. Error bars indicate $\pm \mathrm{SE}$ revealed that percentage cover did not differ significantly between fresh capsules and trocophore stage capsules $(\mathrm{p}=0.671)$, whereas developed capsules were significantly more fouled than both fresh and trocophore stage capsules $(\mathrm{p}<0.001, \mathrm{p}=0.002$, respectively, Fig. 4a).

\section{LIVE/DEAD BacLight bacterial viability assay}

BacLight successfully stained microorganisms on the surface of Dicathais orbita and Sepioteuthis australis egg capsules (Fig. 5). However, the unidentified droplets on the surface of $D$. orbita capsules did not stain with either of these nucleic acid dyes. Control experiments demonstrated that ethanol-killed bacteria gave the expected red fluorescence, while viable cells remained green owing to retention of SYTO9. The egg capsules of both molluscan species were found to have a mixture of live and dead bacterial cells on their surface (Figs. $5 \& 6$ ). The thickness of the biofilm on $S$. australis capsules $(70 \mu \mathrm{m}$, Fig. $5 \mathrm{~b})$ was approximately 4 times thicker than the biofilm on $D$. orbita egg capsules (18 $\mu \mathrm{m}$, Fig. 5a). Consistent with this, the total green fluorescence on the surface of $S$. australis capsules was found to be significantly higher than the green fluorescence emitted from $D$. orbita capsules $(F=193.61$, df $=$
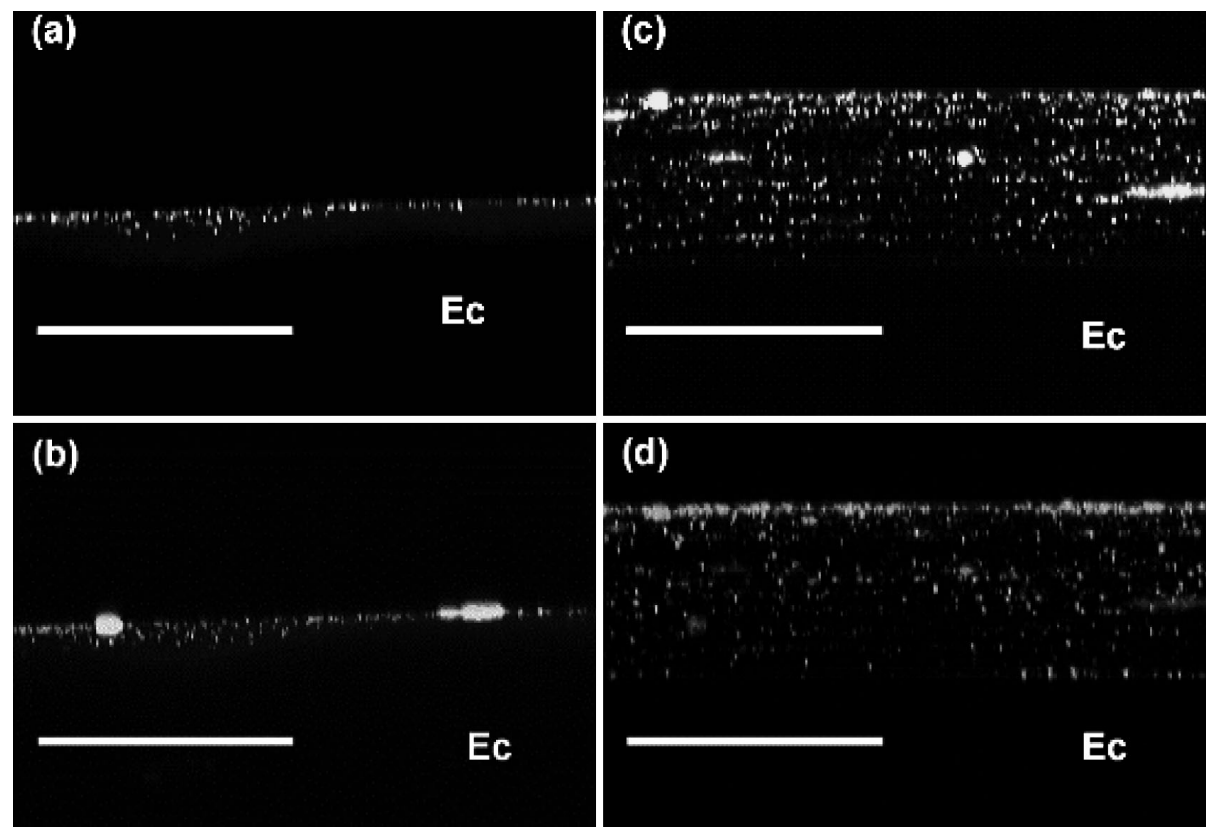

Fig. 5. $x z$ images of molluscan egg capsules stained with LIVE/DEAD BacLight viability stain taken using confocal laser scanning microscopy (CLSM): (a) live biofilm (SYT09 stained) on Dicathais orbita egg capsule; (b) dead biofilm (propidium iodide stained) on D. orbita egg capsule; (c) live biofilm (SYT09 stained) on Sepioteuthis australis egg capsule; (d) dead biofilm (propidium iodide stained) on $S$. australis egg capsule. Images were formed by merging 36 individual scans taken at $2 \mu \mathrm{m}$ intervals. Ec: egg capsule. Scale bars $=60 \mu \mathrm{m}$ 


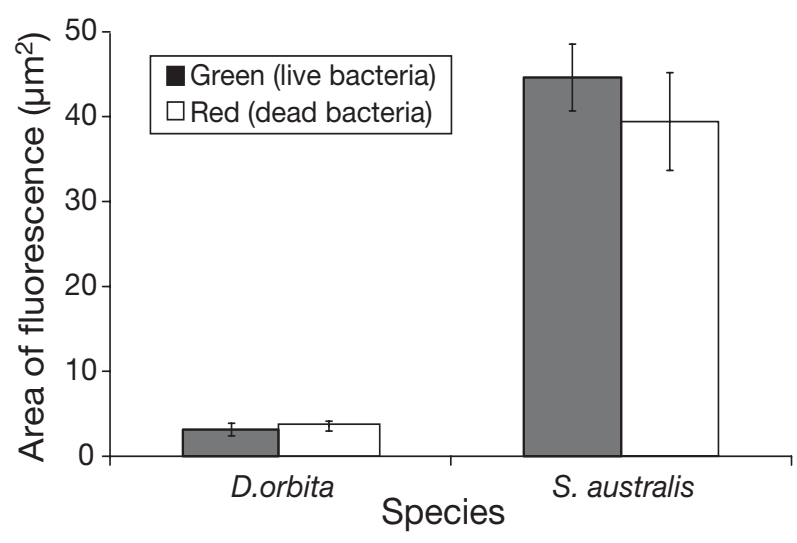

Fig. 6. Dicathais orbita and Sepioteuthis australis. Mean area of green and red fluorescence on the surface of egg capsules after staining with BacLight cell viability stain. Error bars indicate mean $\pm \mathrm{SE}$

$1, \mathrm{p}<0.001)$. Similarly, the total red florescence was significantly higher on $S$. australis than on $D$. orbita egg capsules $(F=75.89$, df $=1, \mathrm{p}<0.001)$.

No significant difference was observed between the amount of live vs. dead microbes for either Sepioteuthis australis $(t=1.747, \mathrm{df}=5, \mathrm{p}=0.141)$ or Dicathais orbita ( $t=0.898, \mathrm{df}=5, \mathrm{p}=0.410$ ) (Fig. 6). However, the proportion of healthy bacteria was slightly higher $(53 \%)$ than that of dead bacteria on surfaces of S. australis, whereas a slightly greater proportion of dead bacteria $(52.5 \%)$ was present on the surface of $D$. orbita egg capsules. Under the CLSM, dead bacteria in association with a string of the unidentified droplets were observed on the egg capsules of $D$. orbita (data not shown). Fluorescence microscopy also revealed an association between dead bacteria and the droplets; however, small colonies of live bacteria were sometimes observed around the droplets as well.

\section{Bacterial attachment}

S91SB was able to survive and attach to egg capsule surfaces within flow chambers. Autofluorescence of egg capsules was observed as blue, which allowed differentiation of GFP-fluorescing bacteria. S91SB did not attach well to the surface of the Dicathais orbita egg capsules (Fig. 7), but some small micro-colonies of bacteria were observed after $24 \mathrm{~h}$. Higher densities of bacteria were occasionally seen attached to the glass slide as a layer of floating GFP-producing bacteria above the surface of the capsule (data not shown). TPLSM images also showed the unidentified droplets on the surface of $D$. orbita egg capsules, but these were not associated with S91SB attachment. In contrast, S91SB was almost always observed to colonize the surface of

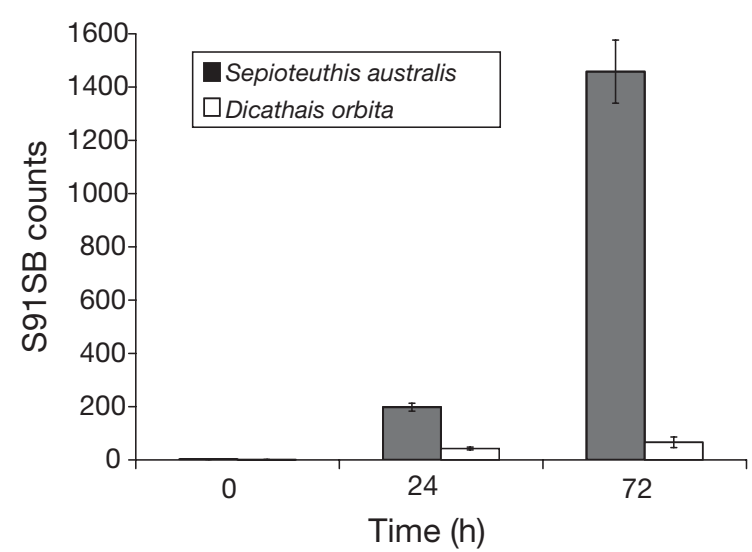

Fig. 7. Dicathais orbita and Sepioteuthis australis. Comparison of Pseudoalteromonas sp. Strain S91SB counts on egg capsules at different time points after exposure in a flow chamber. Error bars indicate mean $\pm \mathrm{SE}$

Sepioteuthis australis egg capsules in high densities (Fig. 7).

The number of S91SB bacteria attaching to the surface of egg capsules from the 2 species was quantified by the area of fluorescence. ANOVA revealed a significant difference among the 3 time points in Dicathais orbita $(F=7.717, \mathrm{df}=2, \mathrm{p}=0.005)$. Post hoc analysis revealed that there were significantly fewer attached $\mathrm{S} 91 \mathrm{SB}$ at $0 \mathrm{~h}$ than at $72 \mathrm{~h}(\mathrm{p}=0.004)$ (Fig. 7). The number of attached S91SB at $24 \mathrm{~h}$ did not differ significantly from that at $0 \mathrm{~h}(\mathrm{p}=0.062)$ or $72 \mathrm{~h}(\mathrm{p}=0.370)$. For Sepioteuthis australis, increasing numbers of S91SB clearly attached to the surface over the $3 \mathrm{~d}$ period (Fig. 7), and significant differences were observed among the time points $(F=151.871$, df $=2$, $\mathrm{p}<0.001)$. However, post hoc analysis revealed that there was no significant difference between 0 and $24 \mathrm{~h}$ $(\mathrm{p}=0.146)$, whereas bacterial load significantly increased after $72 \mathrm{~h}$, with $\mathrm{p}<0.001$ for both the 0 and $24 \mathrm{~h}$ time points. Independent $t$-tests were performed to compare between species at 24 and $72 \mathrm{~h}$. This revealed significantly higher bacterial counts on the surface of $S$. australis than on $D$. orbita egg capsules after both $24 \mathrm{~h}(t=9.765, \mathrm{df}=6.2, \mathrm{p}<0.001)$ and $72 \mathrm{~h}$ $(t=12.45, \mathrm{df}=5.3, \mathrm{p}<0.001)$ in the flow chamber (Fig. 7).

\section{DISCUSSION}

Egg capsules, being sessile in the marine environment, are susceptible to microbial assault (Shields 1990, Benkendorff et al. 2001). Therefore, they require some form of protection against fouling organisms. From this study, it is evident that the egg capsules of Dicathais orbita have anti-fouling defense mechanisms 
that reduce the impacts of microbial foulers on the encapsulated embryos until they are released as pelagic larvae. This was shown by comparatively low microbial densities observed on samples taken directly from the field and after experimental induction of bacterial attachment in the laboratory. By comparison, the eggs of Sepioteuthis australis appear to provide a favourable environment for fouling organisms, but may be more resistant to microbial penetration owing to the overlapping of multiple layers in the capsule wall, which is 15 times thicker than that of the D. orbita capsules (Fig. 1).

As expected from the longer exposure times, the percentage cover of fouling viewed on the egg capsules of both species increased as embryonic development progressed (Fig. 4). Percentage coverage of fouling organisms on the surface was significantly higher in developed capsules than in fresh capsules $(p<0.001)$. A greater range of fouling organisms was observed on the squid egg capsules (e.g. Fig. 3) than on those of Dicathais orbita, including several bacterial morphotypes as well as micro- and macroalgae. The presence of filamentous algae on well-developed Sepioteuthis autralis egg capsules is consistent with independent observations on a Tasmanian population of this species by Steer et al. (2002), who showed that algal fouling promotes synchronized development of $S$. australis egg capsules. The beneficial effects from fouling by photosynthetic algae could include increased oxygen levels for encapsulated embryos during the day (Cohen \& Strathmann 1996), protection from the effects of UV radiation (Biermann et al. 1992) and possibly a reduction in settlement by other fouling organisms, because many algae are known to produce antifouling compounds (Schmitt et al. 1995, Dworjanyn et al. 1999, Maréchal et al. 2004).

The capsules of Sepioteuthis australis are multi-laminated, with layers arranged like a plywood structure (Fig. 1b), which partly accounts for their tough and elastic nature. The multiple layers and overall thickness of these capsules could enable periodic shedding of built-up biofilm, though this was not observed in any of the specimens examined here. Rather, the irregular folds on the surface of $S$. australis capsules (Fig. 3) appear to provide suitable habitat for bacterial settlement (refer to Verran \& Boyd 2001). Field studies on micro-textured surfaces have also revealed that surfaces with $5 \mu \mathrm{m}$ wide ridges favor the settlement of green algae spores (Callow et al. 2002) owing to the valleys and sidewalls of these structures that provide an energetically stable attachment site. The complex structure of $S$. australis egg capsules could facilitate microbial settlement to enable biodegradation of the thick, multi-laminated capsule wall in order to eventually enable the release of the juvenile squids. Unlike
Dicathais orbita capsules, cephalopod egg capsules do not have an escape aperture and the juveniles must therefore break through the wall to be released. Molluscan species that lack escape aperture can typically only emerge once the capsule wall starts to deteriorate (Rivest 1983).

SEM images also provided detailed images of the changes in Dicathais orbita egg capsule morphology over the course of encapsulated development. A thin layer of white crust was seen on the outer surface of fresh capsules (Fig. 2a). This starts to deteriorate after approximately $1 \mathrm{wk}$, exposing the micro-ridged structure of the capsule (Fig. 2b,c). Although the mechanism driving this aging process is unknown, it may simply be eroded under the influence of wave action or currents. Erosion of the periostracum in molluscs (Almeida et al. 1998) has been reported to lead to increasing amounts of boring organisms and settlement of barnacles and algae (Harper \& Skelton 1993, Mao-che et al. 1996, Kaehler 1999, Cerrano et al. 2001). However, after removal of the crust from $D$. orbita capsules, a regular microtopographical feature of ridges and pores was observed throughout the trocophore stage (1 to $3 \mathrm{wk}$ of age) (Fig. 2b). This surface texture is consistent with a physical defense strategy involving minimal contact points for attachment by biofouling organisms. Scardino et al. (2006) provided support for the 'attachment point theory', whereby reduced adhesion strength occurs for fouling organisms that are larger than the scale of microtexture. Nanoridges and pores at a scale suitable for the reduction of microbial attachment were found on the skin of pilot whales Globicephala melas (Baum et al. 2002) and bivalve shells (Scardino et al. 2003). The spacing between ridges on $D$. orbita capsules (1 to $5 \mu \mathrm{m}$, Fig. 2c) compares favorably with the relatively unfouled shells of Mytilus galloprovincialis (1 to $2 \mu \mathrm{m}$, Scardino et al. 2003). Nevertheless, after only $3 \mathrm{wk}, D$. orbita egg capsules maintained in a recirculating seawater system had still become heavily fouled (Fig. 2d).

Egg capsules of Dicathais orbita in the late veliger stage ( $>4 \mathrm{wk}$ ) contained the highest percentage cover of fouling organisms (Fig. 4a) and appeared to be a lot smoother (Fig. 2e,f) than the earlier-stage capsules (Fig. 2a,b). Ablation of the micro-textured outer wall layer was observed under the SEM (Fig. 2e). Mechanical shedding of the biofilm was also reported for the alga Dilsea carnosa during late autumn and early spring (Nylund \& Pavia 2005). The effectiveness of mechanical shedding in limiting surface fouling on $D$. carnosa was suggested to be especially important in temperate regions, where huge variations in fouling organisms arise as a result of seasonal change (Nylund \& Pavia 2005). The egg capsules of D. orbita are deposited on temperate reefs in late spring to summer. 
Thus, shedding of the outer capsule wall may be an effective strategy for preventing biofilm build-up at a key time in their embryonic development when there are heavy microbial loads in the water column.

After shedding of the fouled ridged layer, the surface of Dicathais orbita capsules was found to be relatively free of microbial fouling (Fig. 2e), but areas with increasing densities of bacteria were observed in older capsules, accompanied by large unidentified droplets (Fig. 2f). The droplets appeared to be secreted through pores in the wall as the outer capsule degrades. These droplets were clearly not cellular because they did not stain green or red when incubated with the BacLight nucleic acid probes. They also did not appear to be membrane-bound vesicles, remaining globular rather than dissolving onto the surface. This suggests that they were hydrophobic, which is further supported by the lower densities of droplets observed using standard SEM after critical point drying in ethanol compared with cryostat SEM. Soaking the egg capsules in organic solvents such as dichloromethane was also observed to remove the droplets from the surface (authors' unpubl. data), suggesting that they may contain lipophilic compounds such as the indole dimer tyriverdin, which is a potent bacteriostatic agent previously reported from extracts of $D$. orbita egg capsules (Benkendorff et al. 2000). The droplets were observed to aggregate with bacteria on the capsule surface (Fig. 2f) and fluorescence microscopy revealed they were frequently associated with dead bacteria (data not shown), suggesting that they may have antimicrobial properties. Several species of marine macroalgae have the ability to produce secondary metabolites and release them onto the surface to prevent epibiont settlement (e.g. Ragan \& Glombitza 1986, Clayton \& Ashburner 1994, Dworjanyn et al. 1999). Further studies on the chemical defense of $D$. orbita capsules are clearly required.

Bacterial attachment experiments, undertaken in laboratory flow chambers, further support the idea that Dicathais orbita egg capsules provide a less favorable environment for biofilm formation than do the Sepioteuthis australis capsules. Pseudoalteromonas species are motile marine bacteria commonly found in biofilm communities in temperate Australian waters, and are not known to have any specific interactions with molluscan egg capsules, thus providing a good model system for random biofouling. They secrete chitinases and proteases to break down particulate organic material (POM) (Techkarnjanaruk \& Goodman 1999). Since molluscan egg capsules consist mainly of proteins and carbohydrates (Hunt 1966, Flower et al. 1969, Rapoport \& Shadwick 2002), it was predicted that S91SB would preferentially attach to the egg capsules and utilize them as a carbon source unless the capsules had properties that prevented bacterial attachment. However, S91SB were found to preferentially adhere to the glass coverslips than to $D$. orbita capsules (data not shown). Bacterial attachment was clearly higher at $72 \mathrm{~h}$ than at $0 \mathrm{~h}$; however, there was no significant difference in $D$. orbita capsules between the 24 and $72 \mathrm{~h}$ time points, indicating that the biofilm build-up quickly becomes stagnant (Fig. 7). Conversely, bacterial attachment experiments on $S$. australis showed that S91SB was able to attach to these egg capsules and form a thick layer of biofilm (Fig. 7). A significant increase in S91SB counts at both $24 \mathrm{~h}(\mathrm{p}<0.001)$ and $72 \mathrm{~h}(\mathrm{p}<0.001)$ was observed (Fig. 7), again supporting the notion that antifouling protection is absent from $S$. australis capsules.

The removal and prevention of biofilm formation using physical, mechanical and chemical means has been described in other organisms, but typically these defense mechanisms are studied in isolation. Our present study indicates that the neogastropod Dicathais orbita may use a combination of all these defense mechanisms to defend its egg capsules, including a surface texture not suitable for bacterial attachment, followed by shedding of the outer layer to remove existing microbial colonization and then exudation of unidentified chemical droplets that aggregate and possibly interfere with bacterial growth on the capsules' surface. By comparison, the egg capsules of the cephalopod Sepioteuthis australis had comparatively high loads of live bacteria, and their microtexture appeared to provide good habitat for microbial colonization. Our observations of differences in the surface structure and microbial fouling properties of these 2 molluscan egg capsules contribute to our understanding of antifouling defense mechanisms in marine organisms.

Acknowledgements. We sincerely thank M. Wallwork, J. Terlet and L. Waterhouse (Adelaide Microscopy Unit) for their assistance with this study. We are also grateful to M. Steer (SARDI, Aquatic Sciences) for supplying the Sepioteuthis australis egg capsules. This project was supported by a philanthropic grant, the Australian Research Council, Flinders University and a Flinders University Research Scholarship to K.J.E.

\section{LITERATURE CITED}

Almeida MJ, Machado J, Vieira Coelho MAP, daSilva S, Coimbra J (1998) L-3, 4-dihydroxyphenylalanine (L-DOPA) secreted by oyster (Crassostrea gigas) mantle cells; functional aspects. Comp Biochem Physiol B 120:709-713

Alzieu C (2000) Impact of tributyltin on marine invertebrates. Ecotoxicology 9:71-76

Baum C, Meyer W, Stelzer R, Fleischer LG, Siebers D (2002) Average nanorough skin surface of the pilot whale (Globicephala melas, Delphinidae): considerations on the self- 
cleaning abilities based on nanoroughness. Mar Biol 140: 653-657

Baum C, Simon F, Meyer W, Fleischer LG, Siebers D, Kacza J, Seeger J (2003) Surface properties of the skin of the pilot whale Globicephala melas. Biofouling 19:181-186

Beech IB, Tapper RC, Gubner RJ (2000) Microscopy methods for studying biofilms. In: Evans LV (ed) Biofilms: recent advances in their study and control. Harwood Academic Publishers, Amsterdam, p 51-70

Benkendorff K, Bremner JB, Davies AR (2000) Tyrian purple precursors in the egg masses of the Australian muricid, Dicathais orbita: a possible defensive role. J Chem Ecol 26:1037-1050

Benkendorff K, Davies AR, Bremner JB (2001) Chemical defense in the egg masses of benthic invertebrates: an assessment of antibacterial activity in 39 mollusks and 4 polychaetes. J Invertebr Pathol 78:109-118

Berntsson KM, Jonsson PR, Lejhall M, Gatenholm P (2000) Analysis of behavioural rejection of micro-textured surfaces and implications for recruitment by the barnacle Balanus improvisus. J Exp Mar Biol Ecol 251:59-83

Bers AV, Wahl M (2004) The influence of natural surface microtopographies on fouling. Biofouling 20:43-51

Biermann CH, Schinner GO, Strathmann RR (1992) Influence of solar radiation, microalgal fouling, and current on deposition site and survival of embryos of a dorid nudibranch gastropod. Mar Ecol Prog Ser 86:205-215

Callow ME, Callow JA (2002) Marine biofouling: a sticky problem. Biologist 49:1-5

Callow ME, Jennings AR, Brennan AB, Seegert $\mathrm{CE}$ and 5 others (2002) Microtopographic cues for settlement of zoospores of the green fouling alga Enteromorpha. Biofouling 18:237-245

Cerrano C, Bavestrello G, Calcinai B, Cattaneo-Vietti R, Chiantore M, Guidetti M, Sara A (2001) Bioerosive processes in Antarctic seas. Polar Biol 24:790-792

Chalfie M, Tu Y, Euskirchen G, Ward WW, Prashner DC (1994) Green fluorescent protein as a marker for gene expression. Science 263:802-805

Champ MA (2000) A review of organotin regulatory strategies, pending actions, related costs and benefits. Sci Total Environ 285:21-71

Clayton MN, Ashburner CM (1994) Secretion of phenolic bodies following fertilization in Durvillaea potatorum (Durvillaeales, Phaeophyta). Br J Phycol 29:1-9

Cohen CS, Strathmann RR (1996) Embryos at the edge of tolerance: effects of environment and structure of egg masses on supply of oxygen to embryos. Biol Bull (Woods Hole) 90:8-15

Davis AR, Targett NM, McConnell OJ, Young CM (1989) Epibiosis of marine algae and benthic invertebrates: natural products chemistry and other mechanisms inhibiting settlement and overgrowth. In: Scheuer PJ (ed) Bioorganic marine chemistry, Vol 3. Springer-Verlag, Heidelberg, p 85-114

Delpin MW, McLennan AM, Kolesik P, Goodman AE (2000) Comparison of microcolony formation between Vibrio sp. strain S141 and a flagellum-negative mutant developing biofilms on agar and glass substrata. Biofouling 5:183-193

de Nys R, Steinburg PD (2002) Linking marine biology and biotechnology. Curr Opin Biotechnol 13:244-248

Dworjanyn SA, de Nys R, Steinberg PD (1999) Localisation and surface quantification of secondary metabolites in the red alga Delisea pulchra. Mar Biol 133:727-736

Dyrynda PEJ (1986) Defensive strategies of modular organisms. Phil Trans R Soc Lond B 313:227-243

Engel S, Jensen PR, Fenical W (2002) Chemical ecology of marine microbial defense. J Chem Ecol 28:1971-1985
Everuss K (2004) Competition or cooperation between marine biofilm bacteria recycling POM. BSc Honours thesis, Flinders University of South Australia, Adelaide

Fitch AJ, Kolesik P, Pile AJ, Goodman AE (2002) Plasmid maintenance and localization of Vibrio sp. S141(p519ngfp) cells within monoculture and mixed-species biofilms. Biofouling 18:275-283

Flower NE, Geddes AJ, Rudall KM (1969) Ultrastructure of the fibrous protein from the egg capsules of the whelk Buccinum undatum. J Ultrastruct Res 26:262-273

Goldberg RN, Averbuj A, Cledon M, Luzzatto D, Nudelman NS (2004) Search for triorganotins along the Mar del Plata (Argentina) marine coast: finding of tributylin in egg capsules of a snail Adelomelon brasiliana (Lamarck, 1822) populations showing imposex effects. Appl Organometallic Chem 18:117-123

Hale MS, Mitchell JG (2001) Functional morphology of diatom frustule microstructures: hydrodynamic control of Brownian particle diffusion and advection. Aquat Microb Ecol 24:287-295

Harper EM, Skelton PW (1993) A defensive value of the thickened periostracum in the Mytiloidea. Veliger 36: $36-42$

Hentzer M, Givskov M (2003) Pharmacological inhibition of quorum sensing for the treatment of chronic bacterial infections. J Clin Invest 112:1300-1307

Hunt S (1966) Carbohydrate and amino acid composition of the egg capsule of the whelk, Buccinum undatum. Nature 210:436-437

Kaehler S (1999) Incidence and distribution of phototrophic shell-degrading endoliths of the brown mussel Perna perna. Mar Biol 135:505-514

Kelly SR, Garo E, Jensen PR, Fenical W, Pawlik JR (2005) Effects of Caribbean sponge secondary metabolites on bacterial surface colonization. Aquat Microb Ecol 40: 191-203

Lindquist NJ (2002) Chemical defense of early life stages of benthic marine invertebrates. J Chem Ecol 28:1978-2000

Lord A (1986) Are the contents of egg capsules of the marine gastropod Nucella lapillus (L.) axenic? Am Malacol Bull 4: 201-203

Maki JS (2000) The influence of marine microbes on biofouling. In: Fingermann M, Nagabhushanam R, Thompson MF (eds) Recent advances in marine biotechnology, Vol 3. Biofilms, bioadhesion, corrosion and biofouling. Harwood Academic Publishers, Amsterdam, p 147-171

Mao-che L, Payri C, Le Champion T, Boury-Esnault N, Bezac C, Golubic S (1996) Biodegration of shells of the black pearl oyster Pinctada margaritifera var. cumingii, by microborers and sponges of French Polynesia. Mar Biol 126:509-519

Maréchal JP, Culioli G, Hellio C, Thomas-Guyon H, Callow ME, Clare AS, Ortalo-Magné A (2004) Seasonal variation in antifouling activity of crude extracts of the brown alga Bifurcaria bifurcata (Cystoseiraceae) against cyprids of Balanus amphitrite and the marine bacteria Cobetia marina and Pseudoalteromonas haloplanktis. J Exp Mar Biol Ecol 313:47-62

Nylund GM, Pavia H (2005) Chemical versus mechanical inhibition of fouling in the red alga Dilsea carnosa. Mar Ecol Prog Ser 299:111-121

Östling J, Goodman AE, Kjelleberg S (1991) Behaviour of lnP-1 plasmids and a miniMu transposon in a marine Vibrio sp. S14: isolation of starvation inducible lac operon fusions. FEMS Microbiol Ecol 86:83-94

Pechenik JA (1986) The encapsulation of eggs and embryos by molluscs: an overview. Am Malacol Bull 4:165-172 
Przeslawski R (2004) A review of the effects of environmental stress on embryonic development within intertidal gastropod egg masses. Molluscan Res 24:43-63

Przeslawski R, Benkendorff K (2005) The role of surface fouling in the development of encapsulated gastropod embryos. J Molluscan Stud 71:75-83

Quinn GP, Keough MJ (2002) Experimental design and statistical analysis for biologists. Cambridge University Press, Cambridge

Ragan MA, Glombitza KW (1986) Phlorotannins, brown algal polyphenols. Prog Phycol Res 4:129-241

Rapoport HS, Shadwick RE (2002) Mechanical characterization of an unusual elastic biomaterial from the egg capsule of marine snails (Busycon spp.). Biomacromolecules 3: $42-50$

Rawlings TA (1999) Adaptations to physical stresses in the intertidal zone: the egg capsules of neogastropods molluscs. Am Zool 39:230-243

Rivest BR (1983) Development and the influence of nurse eggs allotment on hatching size in Searlesia dira (Reeve, 1846) (Prosobranchia: Buccinidae). J Exp Mar Biol Ecol 69: $217-241$

Santhana Ramasamy M, Murugan A (2005) Potential antimicrobial activity of marine molluscs from tuticorin, southeast coast of India against 40 biofilm bacteria. J Shellfish Res 24:243-252

Scardino AJ, de Nys R, Ison O, O'Connor W, Steinberg P (2003) Microtopography and antifouling properties of the shell surface of the bivalve molluscs Mytilus galloprovincialis and Pinctada imricata. Biofouling 19:221-230

Scardino AJ, Harvey E, de Nys R (2006) Testing attachment point theory: diatom attachment on microtextured polyimide biomimics. Biofouling 22:55-60

Schmitt TM, Hay ME, Lindquist N (1995) Contraints on chemically mediated coevolution: multiple functions for seaweed secondary metabolites. Ecology 76:107-123

Shields JD (1990) Rhizophydium littoreum on the eggs of Cancer anthonyi: parasite or saprobe? Biol Bull (Woods Hole) 179:201-206

Steer MA, Moltschaniwskyj NA, Gowland FC (2002) Temporal variability in embryonic development and mortality in the southern calamari Sepioteuthis australis: a field assessment. Mar Ecol Prog Ser 243:143-150

Editorial responsibility: Staffan Kjelleberg,

Sydney, New South Wales, Australia
Steinberg PD, De Nys R (2002) Chemical mediation of colonization of seaweed surfaces. J Phycol 38:621-629

Steinberg PD, de Nys R, Kjelleberg S (2001) Chemical mediation of surface colonization. In: McClintock JB, Baker BJ (eds) Marine chemical ecology. CRC Press, Boca Raton, FL, p 355-387

Stickle WB (1973) The reproductive physiology of the intertidal prosobranch Thais lamellose (Gmelin) 1. seasonal changes in the rate of oxygen consumption and body component indexes. Biol Bull (Woods Hole) 144: 511-524

Stretton S, Techkarnjanaruk S, McLennan AM, Goodman AE (1998) Use of green fluorescent protein to tag and investigate gene expression in marine bacteria. Appl Environ Microbiol 64:2554-2559

Techkarnjanaruk S, Goodman AE (1999) Multiple genes involved in chitin degradation from the marine bacterium Pseudoalteromonas sp. strain S91. Microbiology 145: 925-934

Techkarnjanaruk S, Pongpattanakitshote S, Goodman AE (1997) Use of a promoterless lacZ gene insertion to investigate chitinase gene expression in the marine bacterium Pseudoalteromonas sp. S9. Appl Environ Microbiol 63: 2989-2996

Thomason JC, Davenport J, Rogerson A (1994) Antifouling performance of the embryo and eggcase of the dogfish Scyliorhinus canicula. J Mar Biol Assoc UK 74 : $823-836$

Thorson G (1950) Reproductive and larval ecology of marine bottom invertebrates. Biol Rev 25:1-45

Underwood AJ (1997) Experiments in ecology: their logical design and interpretation using analysis of variance. Cambridge University Press, Cambridge

Verran J, Boyd RD (2001) The relationship between substratum surface roughness and microbiological and organic soiling: a review. Biofouling 17:59-71

Whitman WB, Coleman DC, Wiebe WJ (1998) Prokaryotes: the unseen majority. Proc Natl Acad Sci USA 95: $6578-6583$

Yoshida A, Kuramitsu HK (2002) Streptococcus mutans biofilm formation: utilization of a gtfB promoter-green fluorescent protein (PgtfB:gfp) construct to monitor development. Microbiology 148:3385-3394

Submitted: September 8, 2006; Accepted: April 5, 2007

Proofs received from author(s): May 22, 2007 